\title{
Alu-Alu Fusion Sequences Identified at Junction Sites of Copy Number Amplified Regions in Cancer Cell Lines
}

\author{
K. Kitada S. Aikawa S. Aida \\ Kamakura Research Laboratories, Chugai Pharmaceutical Co. Ltd., Kamakura, Japan
}

\section{Key Words}

Alu elements · BFB cycle model · Cancer cell lines · Gene amplification $\cdot$ Inverted repeats

\begin{abstract}
Alu elements are short, 300-bp stretches of DNA and are the most abundant repetitive elements in the human genome. A large number of chromosomal rearrangements mediated by Alu-Alu recombination have been reported in germline cells, but only a few in somatic cells. Cancer development is frequently accompanied by various chromosomal rearrangements including gene amplification. To explore an involvement of Alu-Alu fusion in gene amplification events, we determined 20 junction site sequences of 5 highly amplified regions in 4 cancer cell lines. The amplified regions exhibited a common copy number profile: a stair-like increase with multiple segments, which is implicated in the breakage-fusion-bridge (BFB) cycle-mediated amplification. All of the sequences determined were characterized as head-to-head or tail-to-tail fusion of sequences separated by $1-5 \mathrm{~kb}$ in the genome sequence. Of these, 4 junction site sequences were identified as Alu-Alu fusions between inverted, paired $A$ lu elements with relatively long overlapping sequences of 17, 21, 22, and $24 \mathrm{bp}$. Together with genome mapping data of Alu elements, these findings suggest that when breakages occur at or near inverted, paired Alu ele-
\end{abstract}

ments in the process of BFB cycle-mediated amplification, sequence homology of Alu elements is frequently used to repair the broken ends.

Copyright $\odot 2012$ S. Karger AG, Base

Alu elements are short, $\sim 300-b p$ stretches of DNA and are the most abundant repetitive elements in the human genome, with over 1,000,000 copies [Stenger et al., 2001]. It is well known that homologous recombination between Alu elements generates chromosomal rearrangements including duplications, deletions, and translocations, which contribute to evolutionally expanding genetic diversity in the human genome [Cordaux and Batzer, 2009]. A large number of Alu-Alu recombination-mediated genomic rearrangements in the germline have been identified by comparative genomics [Van de Lagemaat et al., 2005; Sen et al., 2006] and reported as inherited disorders including familial cancers [Batzer and Deininger, 2002; Kolomietz et al., 2002; Franke et al., 2009; Belancio et al., 2010; Konkel and Batzer, 2010; Mitsui et al., 2010; Resta et al., 2010; Boone et al., 2011; Cozar et al., 2011; Kuiper et al., 2011; Borck et al., 2012]. On the other hand, only a few rearrangements mediated by Alu-Alu recombination in somatic cells have been identified. These include deletions in the CAD gene in hepatoma [Hsieh et al., 2005], partial tandem duplications in the $M L L$ gene in acute myeloid 
leukemia [Strout et al., 1998], and tandem duplications in the MYB gene in acute lymphoblastic leukemia [O'Neil et al., 2007]. These are created by recombination between 2 direct $A l u$ repeats and until now no rearrangements created by recombination between 2 inverted $A l u$ repeats have been reported, although such inverted repeats are known to be highly unstable [Gebow et al., 2000].

Gene amplification, defined as an increase in the copy number of specific regions, is commonly observed in human cancer cells and contributes to cancer development by overexpressing oncogenes [Albertson, 2006]. Some amplifications are explained by the breakage-fusion-bridge (BFB) cycle-mediated amplification model [McClintock, 1941; Debatisse and Malfoy, 2005]. In each round of the BFB cycle a broken end of a chromosome is repaired by end-to-end fusion and an inverted duplicated chromosome bearing 2 centromeres is formed. Its junction site sequence exhibits a characteristic structure: a head-to-head or tail-to-tail fusion with a $1-5-\mathrm{kb}$ intervening sequence, as shown in previous studies [Hyrien et al., 1988; Legouy et al., 1989; Okuno et al., 2004; Kitada and Yamasaki, 2007; La Farina et al., 2008]. Such a junction structure is thought to be generated by the non-homologous end joining repair mechanism, which requires no sequence homology. However, it is possible that the structure can also be generated by recombination between inverted, paired Alu elements using their long homology. Indeed, the occurrence of such recombination events has been demonstrated in model constructs [Lobachev et al., 2002; Tanaka et al., 2002; Weinstock et al., 2006; VanHulle, 2007]. In this study, we determined 20 junction site sequences of 5 highly amplified genome regions in 4 cancer cell lines. All the junction sites possessed head-to-head or tail-to-tail fusion sequences with 1-5 kb intervening sequences. Of these, 4 junction sites were identified as Alu-Alu fusion sequences between inverted, paired Alu elements.

\section{Materials and Methods}

\section{Cell Lines}

Two breast cancer cell lines, COLO824 and BT474, a pancreas cancer cell line, AsPC1, and a skin cancer cell line, A431, were used. COLO824 was purchased from DSMZ and the other 3 cell lines were purchased from ATCC.

\section{Array-CGH}

Genomic DNA was extracted from culture cells with a GenomicTip 100 (Qiagen, Valencia, Calif., USA). The procedure for labeling DNA with Cy5-dUTP or Cy3-dUTP and hybridization to microarrays was described in the manufacturer's instructions (Agilent Technologies, Santa Clara, Calif., USA). Human genome
$244 \mathrm{~K}$ standard microarrays and high density custom microarrays with a mean resolution of $125 \mathrm{bp}$ covering the amplified genome regions were used. Fold copy number changes of sample DNA relative to the normal genomic DNA (Promega, Madison, Wisc., USA) were visualized by plotting them in log2 scale against the human genome coordinate using SignalMap software (NimbleGen Systems, Madison, Wisc., USA).

\section{Quantitative PCR}

The quantification of DNA copy numbers was performed using the SYBR green PCR kit in an ABI 7900 system (Applied Biosystems, Foster City, Calif., USA). Primer pairs were designed using Primer3Plus software [Untergasser et al., 2007]. Primer sequences are listed in online suppl. table 1 (for all online suppl. material, see www.karger.com/doi/10.1159/000342885). Realtime qPCR was done twice in $30-\mu$ l reaction mixtures containing 3 different amounts of genomic DNA $(25,50$, and $100 \mathrm{ng})$. Calculated copy numbers are represented as fold change values relative to the normal genomic DNA.

\section{FISH Analysis}

FISH analysis was performed as previously described [Kitada and Yamasaki, 2008]. BAC clones (Invitrogen, Carlsbad, Calif., USA), corresponding to the chromosomal locations of the most amplified segments (between a and b in fig. 1), were labeled with biotin-16-dUTP and used for hybridization. Hybridization signals were detected by avidin-fluorescein (Roche Applied Science, Mannheim, Germany). The BAC clones used were: RP11-795C4 and RP11-458P19 for 6p22.3 of COLO824; RP11-62N3 and CTD3244J20 for 7q22.1 of AsPC1; RP11-116H11 and CTD-2257H21 for 7p11.2 of A431; RP11-395N21 and RP11-201P13 for 9p13.3 of BT474; RP11-1146L22 and RP11-361G24 for 11q13.3 of A431.

\section{Sequence Analysis of Junction Sites}

Based on high density array-CGH data, $\mathrm{PCR}$ primer pairs were designed using Primer3Plus software: one at the end of inverted duplicated regions and another in intervening sequence regions. When array-CGH data did not give information on where intervening regions in the genome sequence were located, primers were designed every $1 \mathrm{~kb}$ (or $0.5 \mathrm{~kb}$ ) to cover the whole sequence region between 2 neighboring segments. Primer3Plus software was used for primer design and LA-PCR (TaKaRa Bio, Otsu, Japan) was used for PCR reactions. The resulting PCR products were directly sequenced with a BigDye Terminator Cycle Sequencing method (Applied Biosystems).

\section{Genome Sequence}

The human genome sequence of NCBI Build 36.1 (http://genome.UCSC.edu/) was used. Alu elements with sequence sizes $>150$ bp were used for the genome mapping.

\section{Results}

Characteristics of Copy Number Amplified Regions in Cancer Cell Lines

We examined 5 amplified regions in 4 cancer cell lines: 6p22.3 in COLO824, 7q22.1 in AsPC1, 7p11.2 in A431, 
9p13.3 in BT474, and 11q13.3 in A431. All amplified regions show a characteristic pattern in the copy number profile: a stair-like increase with multiple segments on one side and a single sharp drop on the other side (fig. 1). Copy number increases with this pattern can be explained by the BFB cycle-mediated gene amplification (online suppl. fig. 1). Junction a, located where the copy number drops sharply in figure 1, would have been generated by repair of the double-strand breakage which initiates the BFB cycle. The amplified regions consisted of 3-5 segments and the most amplified segment (between $a$ and $b$ in fig. 1) has a size of 1,245, 634, 264, 397, and 638 $\mathrm{kb}$ for 6p22.3 (COLO824), 7q22.1 (AsPC1), 7p11.2 (A431), 9p13.3 (BT474), and 11q13.3 (A431), respectively. Fold copy number change of this segment measured by quantitative PCR was 3.7, 14.1, 12.9, 6.4 and 10.7, respectively. Some of these regions have been previously reported as amplified regions [Lin et al., 1984; Merlino et al., 1984; Ullrich et al., 1984; Yoshida et al., 1988; Heidenblad et al., 2004; Shadeo and Lam, 2006].

Using FISH probes prepared from the most amplified segments, localization of amplified regions in metaphase chromosomes was examined. One strong fluorescent signal was observed in COLO824 cells, while 2 strong signals were observed in the other cell lines (fig. 2). In most of the cells of AsPC1, BT474, and A431 (11q13.2), 2 signals with similar intensities were located at the ends of 2 chromosomes, which appeared identical in shape. These 2 chromosomes are presumed to have been generated by duplication of 1 chromosome carrying the regional amplification. Near-tetraploidy of BT474 and near-triploidy of A431 would be related to the duplication. In A431 (7p11.2), 2 signals with different intensities appeared in different-shaped chromosomes, suggesting that these 2 regional amplifications occurred independently. Further study, especially individual analysis of individually isolated chromosomes, as described before [Kitada et al., 2011], is needed for detailed characterization.

\section{Determination of Junction Site Sequences}

To determine junction site sequences, DNA fragments containing junction sites were obtained by PCR-based amplification and sequenced. In total, 20 junction site sequences were determined and the results are summarized in table 1 . Junction sites $\mathrm{a}, \mathrm{b}, \mathrm{c}, \mathrm{d}$, and e correspond to junctions $\mathrm{a}, \mathrm{b}, \mathrm{c}, \mathrm{d}$, and e in figure 1 .

The sequence structure of junction site A431-7-b is complicated. An inverted duplication was formed by head-to-head fusion at sequence positions 55,204,390 and 55,208,110 and 3 short sequences were sandwiched:

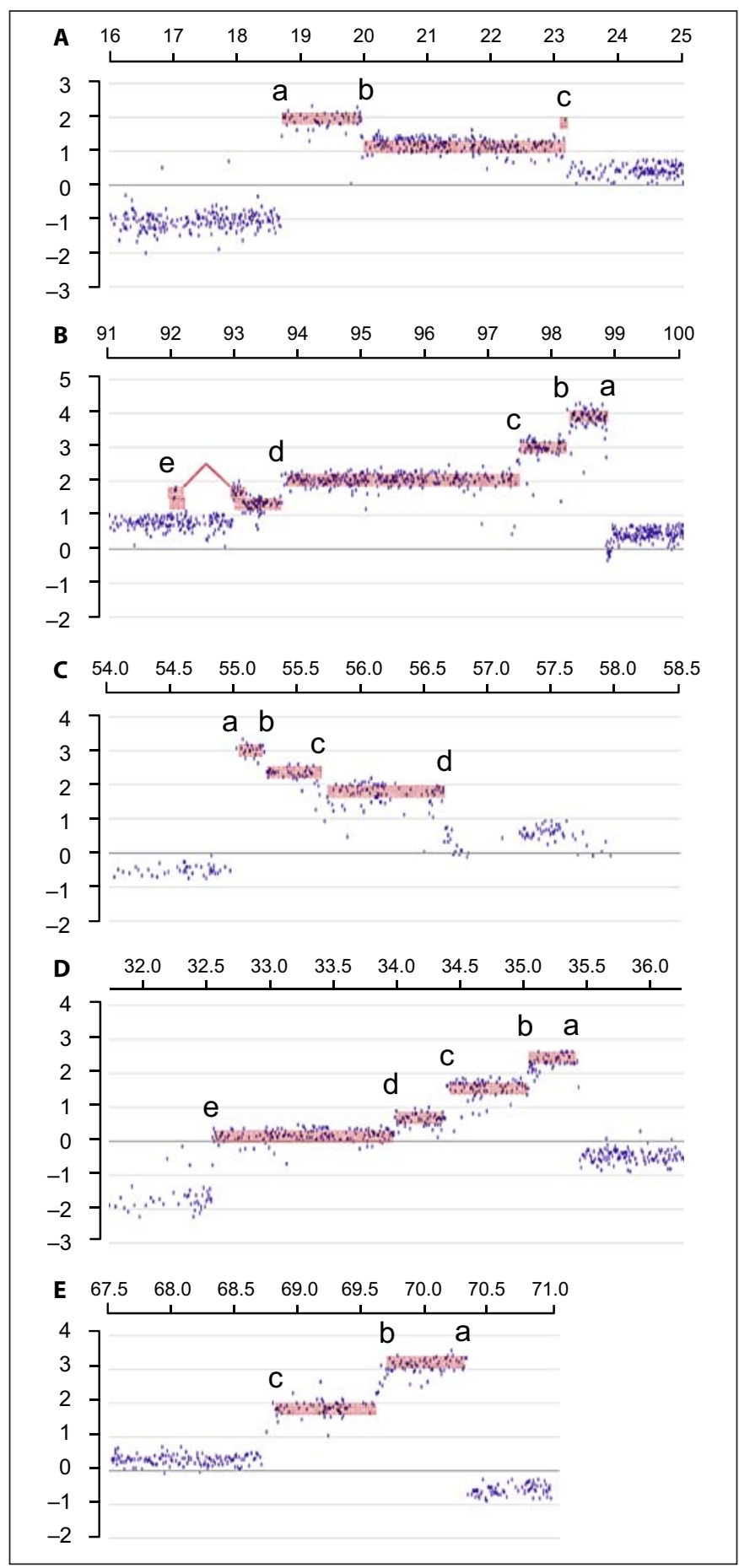

Fig. 1. Copy number amplifications in cancer cell lines. Five amplified genomic regions in 4 cancer cell lines were selected: A 6p22.3 in COLO824; B 7q22.1 in AsPC1; C 7p11.2 in A431; D 9p13.3 in BT474; E 11q13.3 in A431. Copy number profiles analyzed by array-CGH. Fold change values of genomic DNA of cell lines relative to the normal genomic DNA are visualized by plotting them in $\log 2$ scale (vertical axis) against the sequence coordinate in $\mathrm{Mb}$ scale (horizontal axis). Each amplified segment is marked by a red bar with junction IDs (a, b, c, d, and e). 

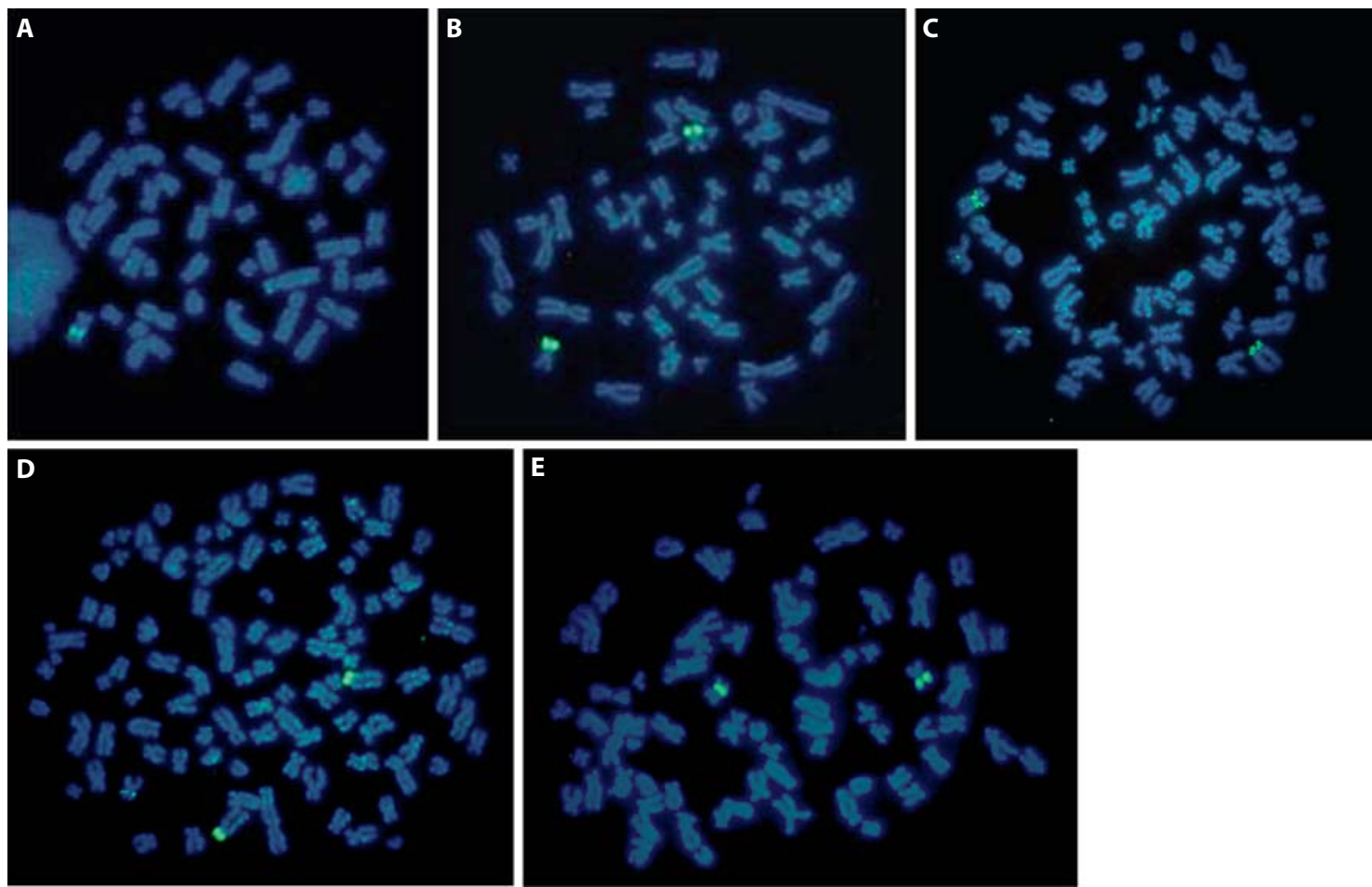

Fig. 2. Chromosomal locations of amplified regions. FISH probes, which were generated by fluorescently labeling BAC clone DNA derived from genomic regions of the most amplified segments (between a and b in fig. 1), were hybridized to spreads of metaphase chromosomes. Hybridized signals were visualized as green dots in the DAPI-stained chromosomes. A 6p22.3 in COLO824; B 7q22.1 in AsPC1; C 7p11.2 in A431; D 9p13.3 in BT474; E 11q13.3 in A431.

$775 \mathrm{bp}$ from chromosome 4 and 79 and $345 \mathrm{bp}$ from chromosome 7 (online suppl. fig. 2A). Except for A431-7-b, all 19 junction sites showed a common structure: head-tohead or tail-to-tail fusions with intervening sequences with a mean size of $2.4 \mathrm{~kb}$, ranging from 0.2 to $8.8 \mathrm{~kb}$. Short overlapping sequences of $1-4 \mathrm{bp}$ were found in 14 junction site sequences and an insertion of $10 \mathrm{bp}$ was found in 1 junction site, BT474-9-c. For the remaining 4 junction sites, relatively longer overlapping sequences were identified: 24, 22, 17, and $21 \mathrm{bp}$, for AsPC1-7-c, AsPC1-7-e, BT474-9-d, and A431-11-b, respectively. Sequence alignment revealed that these overlapping sequences came from identical sequences between paired, inverted Alu elements (fig. 3). Various Alu subfamilies were combined: AluSc-AluY (separated by $2.9 \mathrm{~kb}$ ) for AsPC1-7-c; AluSg-AluSx (2.5 kb) for AsPC1-7-e; AluJoAluSx (1.6 kb) for BT474-9-d; AluSx-AluSx (3.4 kb) for A431-11-b.

Alu elements around breakpoints of junction sites were mapped on the genome sequence (online suppl. fig. 3). The numbers of Alu elements which resided between 2 breakpoints joined at each junction site are summarized in table 1. Out of all 19 junction sites (after removal of junction site A431-7-b due to the complicated structure), 9 contained no Alu elements and 4 contained 1 or $2 \mathrm{Alu}$ elements with the same orientation. The remaining 6 junction sites contained more than 1 pair of inverted $A l u$ elements. Of these, 4 junction sites were found as fusions of inverted, paired Alu elements (fig. 3).

\section{Discussion}

We have determined 20 junction site sequences of 5 amplified regions in 4 cancer cell lines. We believe these amplified regions are likely to have been amplified by the repetition of $\mathrm{BFB}$ cycles from the following findings. First, in metaphase FISH analysis, amplified regions appeared as homogeneously staining regions. Second, in array-CGH analysis, the amplified regions displayed a 
Table 1. Junction site structures

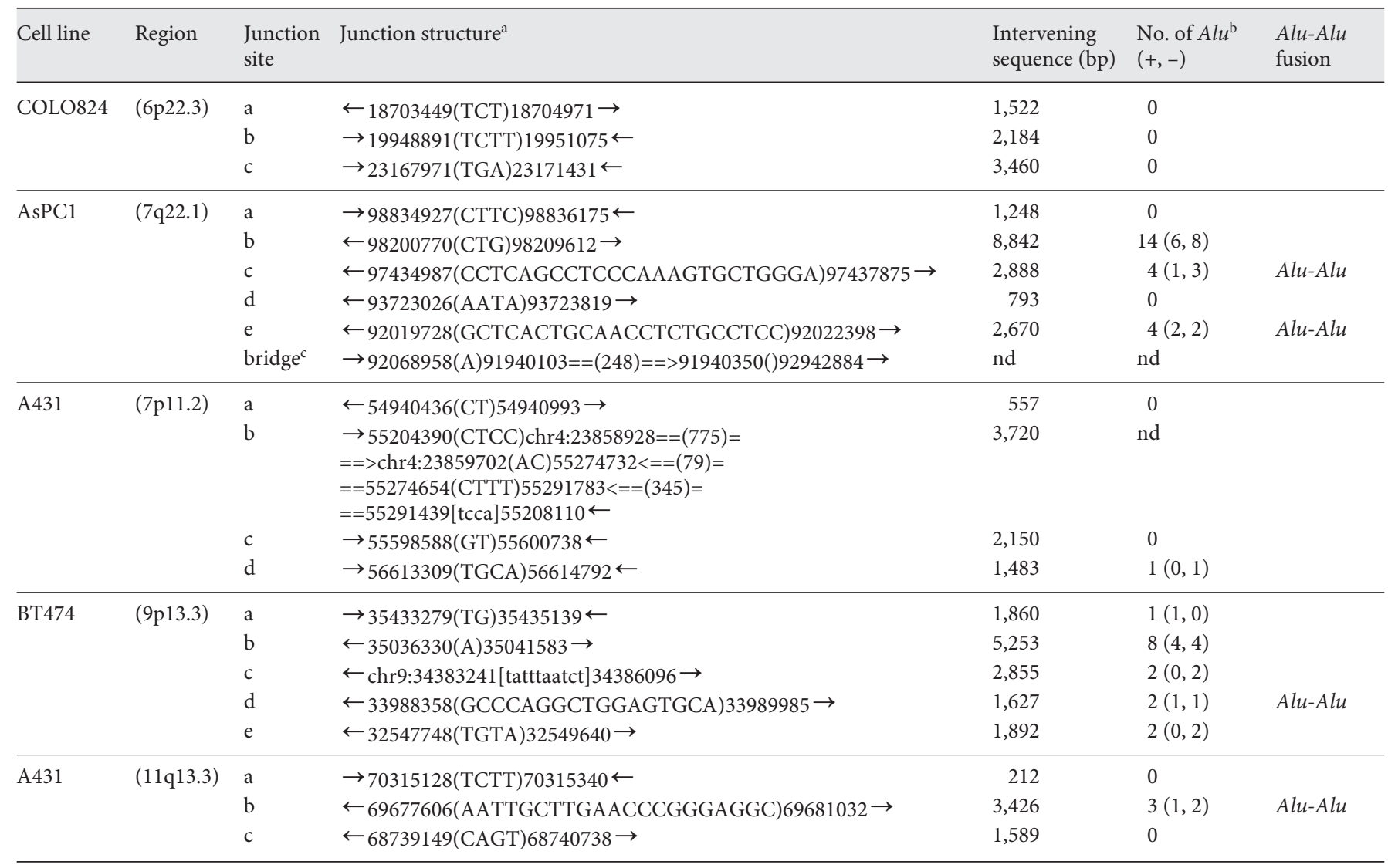

nd $=$ No data.

a Junction structures are represented as sequence positions and directions (numbers and arrows) with overlapping nucleotides (upper case letters), inserted nucleotides [lower case letters], or no insertions ().

b The numbers of Alu elements between 2 breakpoints which are joined at each junction site are indicated. Alu elements on the plus (+) or minus (-) strand are shown in parentheses.

${ }^{c}$ Two separated sequence positions are bridged with a small sequence stretch (248 bp), resulting in a deletion of $\sim 900 \mathrm{~kb}$. (See online suppl. fig. $2 \mathrm{~B}$ for the structure).

characteristic copy number profile; that is, a stair-like increase with multiple segments on one side and a single sharp drop on the other side. Third, in sequence analysis, junction sites of the amplified regions exhibited a characteristic structure such as head-to-head or tail-to-tail fusion with a 1-5 kb intervening sequence at the center, accompanied by overlapping sequences. These junction structures are known to be generated by end-to-end fusion of broken chromosomes using a non-homologous end joining repair mechanism [Okuno et al., 2004; Hefferin and Tomkinson, 2005].

Out of the 20 junction sites, sequences of 4 junction sites, AsPC-7-c, AsPC-7-e, BT474-9-d, and A431-11-b, were found as fusion sequences between inverted, paired Alu elements, which are separated by $1-3 \mathrm{~kb}$ in the ge- nome sequence. These fusion sequences contain relatively long overlapping sequences of $17,21,22$, and $24 \mathrm{bp}$, identical in both Alu elements, when compared with other junction site sequences, which contain $1-8$ bp overlapping sequences. This information on the sequences suggests that the junctions may be generated by the single strand annealing pathway of recombination, an alternative repair mechanism using sequence homology between inverted, paired Alu elements. The occurrence of such events has been previously demonstrated in model constructs [Lobachev et al., 2002; Tanaka et al., 2002; VanHulle et al., 2007]. With a triggering double strand break at or near inverted, paired Alu elements, one Alu sequence can anneal to the other inverted Alu sequence on the same strand (before DNA replication) or a differ- 
Fig. 3. Alu-Alu fusions at junction sites. Four junction sites in 3 amplified regions are shown. A AsPC1-7-c; B AsPC1-7-e; C BT474-9-d; D A431-11-b. Upper panels: Genome map of Alu elements and breakpoints. Alu elements in the plus orientation (solid boxes above the line) and in the minus orientation (solid boxes below the line) are mapped on the genome sequence. The 2 breakpoints, which are joined at each junction site, are mapped on the genome sequence using red and blue colored bars. The sequence between red and blue bars indicates an intervening sequence and the sequence at the side of a redmarked breakpoint is over hanging. Lower panels: Sequence alignment of $2 \mathrm{Alu}$ elements fused at the junction site. Underlined sequences represent junction site sequences and sequences in red represent identical sequences in both Alu elements, within which the exact fusion point resides. Numbering indicates the sequence positions on the genome sequence.

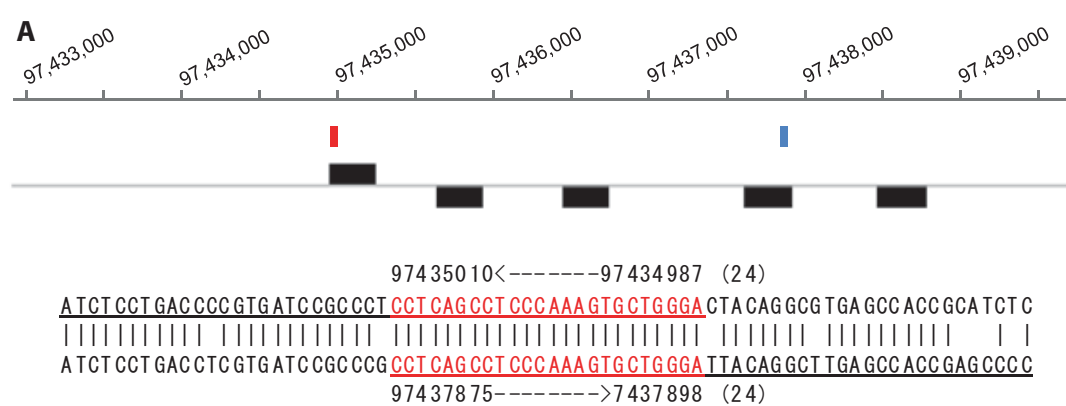

B

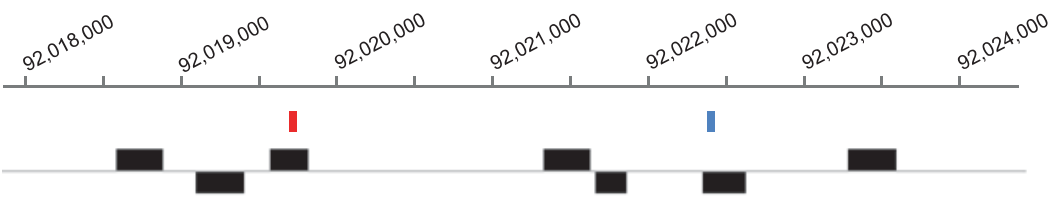

$92019749<---92019728$ (22)

GCTGGAGGGCAGT GGCGCTATC TCGGCT CAC TGCAAC CTC TGC CTCCCGGGT TCA AGC AAT TCT CCT GCC TC

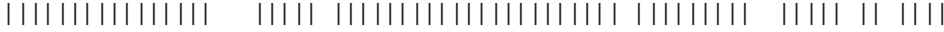
GCTGGAGGGC AGT GGC ATG ATC TCA GCT CAC TGC AAC CTC TGC CTC CTGGGT TCAAGT GAT TCT ACT ACC TC 92022398----->92022419 (22)

C
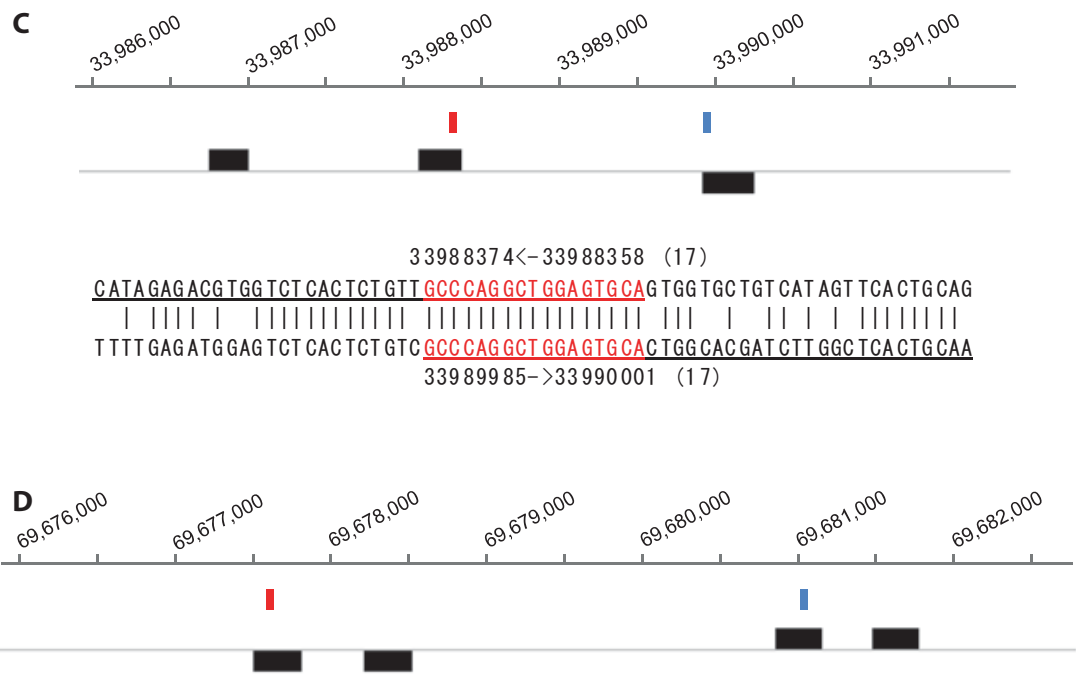

$69677626<----69677606 \quad(21)$

GGTA CCC GGG AGG CTGAGG CAT GAGAAT TGC TTGAAC CCG GGA GGC GGG GGT TGC AGT GAG CCG AGA TCA T

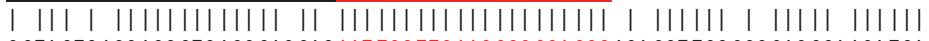
GCTACTCAGG AGG CTGAGG CAG GACAAT TGC TTGAAC CCG GGAGGCAGAGGT TGCGGGGAGCCAAGA TCAC $69681032--->69681052(21)$ ent strand (after DNA replication) and an inverted duplicated chromosome with a head-to-head or tail-to-tail fusion junction is formed. In addition, the Alu mapping data revealed that 6 junctions, containing at least 1 pair of inverted elements, have the potential to make an in- verted Alu-Alu fusion. Out of these junctions, 4 were found as inverted Alu-Alu fusions. The high occurrence rate (4/6) of such a fusion event suggests the possibility that, if the breaks occur at or near inverted, paired Alu elements, broken ends induced by the process of BFB cy- 
cle-mediated amplification could be mainly repaired by single strand annealing using sequence homology of Alu elements. On the other hand, in this study no Alu-Alu fusion was found at the junction sites created by the first round of BFB cycles (junctions a in fig. 1 and junction sites a in table 1). This suggests that Alu-mediated repair does not participate in the initiation of the BFB cycle. However, the lack of Alu-Alu fusion could also be attributed to the absence of Alu elements near the breakpoint sites and, therefore, further study is required before conclusions as to relationship between the $A l u$-mediated repair and the initiation of the BFB cycle can be drawn.

So far, Alu-Alu fusion-mediated chromosomal rearrangements reported in somatic cells are very few compared with those in human germline cells [Kolomietz et al., 2002; Belancio et al., 2010; Konkel and Batzer, 2010; Deininger, 2011], and those that have been reported are tandem duplications of $M L L$ [Strout et al., 1998] and $M Y B$ [O'Neil et al., 2007] and deletions of CAD [Hsieh et al., 2005]. However, the current study shows the possibility that even in somatic cells the Alu-Alu fusion occurs in a type of chromosomal rearrangement. Recently, by us- ing a genome-wide searching assay, DNA fragments with palindromic structures have been isolated from cancer genomes and then mapped to the genome sequence. The mapping shows that such fragments are not randomly distributed in the cancer genomes and are often located in clusters, especially at the boundaries of amplified regions [Guenthoer et al., 2012]. It would be interesting to examine whether some of these fragments contain inverted $A l u$-Alu fusion sequences. In addition, by genomewide sequence analysis using the next generation sequencing technology, 3 inverted Alu-Alu fusion sequences have been identified in a breast cancer cell line [Bignell et al., 2007]. Thus, further studies, especially genomewide sequence analysis, will identify novel Alu-Alu fusions occurring in somatic cells and will further our understanding of the relationship between such fusions and somatic rearrangements.

\section{Acknowledgement}

We would like to thank Ikuko Matsuo and Noriko Maki for DNA sequencing.

\section{References}

Albertson DG: Gene amplification in cancer. Cozar M, Bembi B, Dominissini S, Zampieri S, Trends Genet 22:447-455 (2006).

Batzer MA, Deininger PL: Alu repeats and human genomic diversity. Nat Rev Genet 3: 370-379 (2002).

Belancio VP, Roy-Engel AM, Deininger PL: All y'all need to know 'bout retroelements in cancer. Semin Cancer Biol 20:200-210 (2010).

Bignell GR, Santarius T, Pole JC, Butler AP, Perry J, et al: Architectures of somatic genomic rearrangement in human cancer amplicons at sequence-level resolution. Genome Res 17: 1296-1303 (2007).

Boone PM, Liu P, Zhang F, Carvalho CM, Towne $\mathrm{CF}$, et al: Alu-specific microhomology-mediated deletion of the final exon of SPAST in three unrelated subjects with hereditary spastic paraplegia. Genet Med 13:582-592 (2011).

Borck G, Kakar N, Hoch J, Friedrich K, Freudenberg J, et al: An Alu repeat-mediated genomic GCNT2 deletion underlies congenital cataracts and adult i blood group. Hum Genet 131:209-216 (2012).

Cordaux R, Batzer MA: The impact of retrotransposons on human genome evolution. Nat Rev Genet 10:691-703 (2009). Vilageliu L, et al: Molecular characterization of a new deletion of the GBA1 gene due to an inter Alu recombination event. Mol Genet Metab 102:226-228 (2011).

Debatisse M, Malfoy B: Gene amplification mechanisms, in Nigg EA (ed): Genome Instability in Cancer Development, pp 343 361 (Springer, New York 2005).

eininger P: Alu elements: know the SINEs. Genome Biol 12:236 (2011).

Franke G, Bausch B, Hoffmann MM, Cybulla M, Wilhelm C, et al: Alu-Alu recombination underlies the vast majority of large VHL germline deletions: molecular characterization and genotype-phenotype correlations in VHL patients. Hum Mutat 30:776-786 (2009).

Gebow D, Miselis N, Liber HL: Homologous and nonhomologous recombination resulting in deletion: effects of p53 status, microhomology, and repetitive DNA length and orientation. Mol Cell Biol 20:4028-4035 (2000).

Guenthoer J, Diede SJ, Tanaka H, Chai X, Hsu L, et al: Assessment of palindromes as platforms for DNA amplification in breast cancer. Genome Res 22:232-245 (2012).

Hefferin ML, Tomkinson AE: Mechanism of DNA double-strand break repair by non-homologous end joining. DNA Repair 4:639648 (2005)
Heidenblad M, Schoenmakers EF, Jonson T, Gorunova L, Veltman JA, et al: Genome-wide array-based comparative genomic hybridization reveals multiple amplification targets and novel homozygous deletions in pancreatic carcinoma cell lines. Cancer Res 64: 3052-3059 (2004).

Hsieh SY, Chen WY, Yeh TS, Sheen IS, Huang SF: High-frequency Alu-mediated genomic recombination/deletion within the caspaseactivated DNase gene in human hepatoma. Oncogene 24:6584-6589 (2005).

Hyrien O, Debatisse M, Buttin G, de Saint Vincent BR: The multicopy appearance of a large inverted duplication and the sequence at the inversion joint suggest a new model for gene amplification. EMBO J 7:407-417 (1988).

Kitada K, Yamasaki T: The MDR1/ABCB1 regional amplification in large inverted repeats with asymmetric sequences and microhomologies at the junction sites. Cancer Genet Cytogenet 178:120-127 (2007).

Kitada K, Yamasaki T: The complicated copy number alteration in chromosome 7 of a lung cancer cell line is explained by a model based on repeated breakage-fusion-bridge cycles. Cancer Genet Cytogenet 185:11-19 (2008). 
-Kitada K, Taima A, Ogasawara K, Metsugi S, Aikawa S: Chromosome-specific segmentation revealed by structural analysis of individually isolated chromosomes. Genes Chromosomes Cancer 50:217-227 (2011).

Kolomietz E, Meyn MS, Pandita A, Squire JA: The role of Alu repeat clusters as mediators of recurrent chromosomal aberrations in tumors. Genes Chromosomes Cancer 35:97112 (2002)

Konkel MK, Batzer MA: A mobile threat to genome stability: the impact of non-LTR retrotransposons upon the human genome. Semin Cancer Biol 20:211-21 (2010).

Kuiper RP, Vissers LE, Venkatachalam R, Bodmer D, Hoenselaar E, et al: Recurrence and variability of germline EPCAM deletions in Lynch syndrome. Hum Mutat 32:407-414 (2011).

La Farina M, Bellavia M, Tagliavia M, Eterno V, Colomba P, et al: Two distinct amplification events of the $c-m y c$ locus in a colorectal tumour. J Cell Physiol 217:34-39 (2008).

- Legouy E, Fossar N, Lhomond G, Brison O: Structure of four amplified DNA novel joints. Somat Cell Mol Genet 15:309-320 (1989).

- Lin CR, Chen WS, Kruiger W, Stolarsky LS, Weber W, et al: Expression cloning of human EGF receptor complementary DNA: gene amplification and three related messenger RNA products in A431 cells. Science 224: 843-848 (1984).

Lobachev KS, Gordenin DA, Resnick MA: The Mre11 complex is required for repair of hairpin-capped double-strand breaks and prevention of chromosome rearrangements. Cell 108:183-193 (2002).

McClintock B: The stability of broken ends of chromosomes in Zea mays. Genetics 26:234282 (1941).
Merlino GT, Xu YH, Ishii S, Clark AJ, Semba K, et al: Amplification and enhanced expression of the epidermal growth factor receptor gene in A431 human carcinoma cells. Science 224:417-419 (1984).

-Mitsui J, Takahashi Y, Goto J, Tomiyama H, Ishikawa S, et al: Mechanisms of genomic instabilities underlying two common fragile-siteassociated loci, PARK2 and DMD, in germ cell and cancer cell lines. Am J Hum Genet 87:75-89 (2010).

Okuno Y, Hahn PJ, Gilbert DM: Structure of a palindromic amplicon junction implicates microhomology-mediated end joining as a mechanism of sister chromatid fusion during gene amplification. Nucleic Acids Res 32: 749-756 (2004).

O’Neil J, Tchinda J, Gutierrez A, Moreau L, Maser RS, et al: Alu elements mediate MYB gene tandem duplication in human T-ALL. J Exp Med 204:3059-3066 (2007).

- Resta N, Giorda R, Bagnulo R, Beri S, Della Mina E, et al: Breakpoint determination of 15 large deletions in Peutz-Jeghers subjects. Hum Genet 128:373-382 (2010)

-Sen SK, Han K, Wang J, Lee J, Wang H, et al: Human genomic deletions mediated by recombination between Alu elements. Am J Hum Genet 79:41-53 (2006).

Shadeo A, Lam WL: Comprehensive copy number profiles of breast cancer cell model genomes. Breast Cancer Res 8:R9 (2006).

-Stenger JE, Lobachev KS, Gordenin D, Darden TA, Jurka J, et al: Biased distribution of inverted and direct Alus in the human genome: implications for insertion, exclusion, and genome stability. Genome Res 11:12-27 (2001).

Strout MP, Marcucci G, Bloomfield CD, Caligiuri MA: The partial tandem duplication of $A L L 1$ (MLL) is consistently generated by Alumediated homologous recombination in acute myeloid leukemia. Proc Natl Acad Sci USA 95:2390-2395 (1998).
Tanaka H, Tapscott SJ, Trask BJ, Yao MC: Short inverted repeats initiate gene amplification through the formation of a large DNA palindrome in mammalian cells. Proc Natl Acad Sci USA 99:8772-8777 (2002).

- Ullrich A, Coussens L, Hayflick JS, Dull TJ, Gray A, et al: Human epidermal growth factor receptor CDNA sequence and aberrant expression of the amplified gene in A431 epidermoid carcinoma cells. Nature 309:418-425 (1984).

-Untergasser A, Nijveen H, Rao X, Bisseling T, Geurts R, et al: Primer3Plus, an enhanced web interface to Primer3. Nucleic Acids Res 35:W71-74 (2007).

-Van de Lagemaat LN, Gagnier L, Medstrand P, Mager DL: Genomic deletions and precise removal of transposable elements mediated by short identical DNA segments in primates. Genome Res 15:1243-1249 (2005).

- VanHulle K, Lemoine FJ, Narayanan V, Downing $\mathrm{B}$, Hull $\mathrm{K}$, et al: Inverted DNA repeats channel repair of distant double-strand breaks into chromatid fusions and chromosomal rearrangements. Mol Cell Biol 27: 2601-2614 (2007).

Weinstock DM, Richardson CA, Elliott B, Jasin M: Modeling oncogenic translocations: distinct roles for double-strand break repair pathways in translocation formation in mammalian cells. DNA Repair 5:1065-1074 (2006).

-Yoshida MC, Wada M, Satoh H, Yoshida T, Sakamoto $\mathrm{H}$, et al: Human HST1 (HSTF1) gene maps to chromosome band 11q13 and coamplifies with the INT2 gene in human cancer. Proc Natl Acad Sci USA 85:4861-4864 (1988). 